\title{
Validation of the existence of genuine Empty follicle syndrome, versus false empty follicle syndrome to make definitive decisions in cases where recurrent IVF failure encountered secondary to absence of oocytes on ovum pick up-a short communication
}

\begin{abstract}
Aim: Worldwide a big altercation exists with regards to the actual existence of the term " Empty follicle syndrome"(EFS), so much so that certain big authorities in the field have been believing that true EFS does not exist. Basically EFS is a syndrome when no functionally intact oocyte get retrieved when attempting an oocyte pick up (OPU) for a successful in vitro fertilization (IVF), however such patients encounter recurrent IVF failures. Since it is has become a big problem for the treating reproductive endocrinologist, besides the patient encountering recurrent IVF failures, it has become essential to differentiate the true EFS alias genuine Empty follicle syndrome (gEFS) from what is labeled today as the false empty follicle syndrome (fEFS). In view of the recently documented presence of mutations, gEFS got verified and appears to silence this biggest conflict that arose secondary to the existence of a false empty follicle syndrome (fEFS), where one could manage to get successful IVF outcomes subsequent to repeated hCG injections/ gonadotropin releasing hormone (GnRH) agonist in addition to pregnancy, with lot of clinicians believing there is no true term like EFS.

Methods: Recently Yang et al., performed a study In tertiary a university based reproductive center in China that was comprised of a big cohort of patients that presented with gEFS, Genetic evaluation was conducted on 35 non correlated infertile patients who went through 16 failed IVF cycles in addition to oocyte degeneration, besides the subjects got a diagnosis of possessing a particular kind of EFS- cumulus oocytes complexes (COC's) but possessed oocytes that were undergoing degeneration, with the utilization of whole-exome sequencing along with targeted Sanger sequencing.
\end{abstract}

Results: Yang et al., found 22 innovative genetic variant of zona pellucida (ZP), genes in 18 subjects, that were inclusive of 20 variants in ZP 1 gene, 2 in ZP 2 gene in addition to 1 recurring variant in ZP3 gene that had been earlier documented. The homogenous / compound heterogenous ZP 1 mutations were inherited in an autosomal recessive manner, while the heterogenous variants of ZP 2 as well as ZP3 genes possessed an autosomal dominant manner of inheritance.

Conclusions: These mutations were anticipated to be harmful in silico along with got further experimentally corroborated to be functionally null dependent on their ectopic expression in vitro. Thus with this further evidence that has been recently provided with regards to the existence of genuine Empty follicle syndrome (gEFS), it is significant for the youngsters to realize if they encounter similar cases after trial of rescue hCG injections / GnRH) agonist, not to further keep waiting, but evaluate further with regards to the existence of mutations for Zona Pellucida (ZP), ZP 1, ZP2 as well as ZP3 genes mutations, or LH/ chorionic gonadotropins receptor (LHCGR) gene mutation without subjecting the patient to repeated IVF, with her psychological as well as financial health in mind.

Keywords: gEFS, fEFS, LHCGR, gene mutation, ZP mutations
Volume 7 Issue 4 - 202I

\author{
Kulvinder Kochar Kaur,' Gautam Allahbadia, ${ }^{2}$ \\ Mandeep Singh ${ }^{3}$ \\ 'Dr Kulvinder Kaur Centre for Human Reproduction, India \\ 2Ex-Rotunda-A Centre for Human Reproduction, India \\ ${ }^{3}$ Consultant Neurologist, Swami Satyanand Hospital, India
}

Correspondence: Kulvinder Kochar Kaur, Scientific Director, Dr Kulvinder Kaur Centre for Human Reproduction 72I, G.T.B. Nagar, Jalandhar-1 4400I, Punjab, India, Tel 91-181-9501358180, Email mkulvinder.dr@gmail.com

Received: August 14, 2021 | Published: August 31, 2021

\section{Short communication}

For doing a successful in vitro fertilization (IVF) the basic requirement is retrieving a functionally intact oocyte. Clinically we usually attain this by delivering a bolus of recombinant $\beta$ human chorionic Gonadotropins (HCG) bolus or urinary HCG or gonadotropin releasing hormone $(\mathrm{GnRH})$ agonist, that results in Luteinizing hormone (LH) surge, which is followed by ovulation. Nevertheless, In occasional cases, no oocytes get obtained despite the existence of enough ovarian stimulation, that gets checked by the amounts of HCG along with estradiol (E2) amounts, besides careful oocyte pick up (OPU). This becomes a very tough situation for the patient who is undergoing IVF, besides for the treating physician, a situation that is also termed as genuine empty follicle syndrome (gEFS), that got $1^{\text {st }}$ documented by Coulam et al. in $1986 .{ }^{1}$ In contrast to the false empty follicle syndrome (fEFS), that is a disorder where no oocytes get obtained secondary to inadequate circulating HCG amounts, which might occur due to improper delivery of HCG or bioavailability of 
the same. ${ }^{2,3}$ Hence As per the $\beta$ HCG concentrations on the day of the OPU, EFS got classified as fEFS in addition to gEGS. ${ }^{2,3}$ During the last few years, the separation among gEFS in addition to $\mathrm{fEFS}^{2,3}$ continues to be a topic on which a lot of altercation continues in view of a lot of clinicians observed intact oocytes as well as a favourable pregnancy following rescue stimulation of the ovary following delivery of HCG/ GnRH agonist again subsequently to a failed earlier try. ${ }^{4}$ Thus fEFS subjects possess the probability of attaining a successful pregnancy following the redelivery of $\mathrm{HCG}$ or repeating OPU..$^{5-7}$ Comparative to fEFS the incidence, of gEGS is markedly low. $2,3,8,9$

In normal physiological conditions, ovulation represents a very complicated event over which we still have not gained full insight. ${ }^{10}$ About $36 \mathrm{~h}$ prior to, ovulation, great concentrations of E2 get generated within the growing follicles, shifting suddenly(in the currently believed model of ovulation induction) from its hampering function towards the one of stimulation of liberation of GnRH from the hypothalamus, resulting in an LH surge which triggers the ultimate rupture of the follicle it has got documented that ay aberrations that have an impact on this event can result In failure of subsequent ovulation. Like bad ovarian reaction, premature ovulation, inherent deficits of biological action in addition to impairment in folliculogenesis have all been documented to result in fEFS in case of patients. In this context, a long gap among delivery of hCG along with oocyte pick up, a rescue hCG injection to an earlier failure, or utilization of dual triggering with hCG along with GnRH agonist, actually aid in getting intact oocytes, that aid In successful pregnancies in addition to live birth rates( $\mathrm{LBR})$. $^{2,3}$

Conversely it can be posited that genetic aberrations taking place in folliculogenesis or ovulation pathway might generally damage oocyte pick up subsequent to ovulation induction with hormones. Factually, LH primarily liberated by follicles as well as hCG generated by the placenta are structurally in addition to functionally akin to each other, besides possessing identical receptors in vivo, along with actually a lot of various mutations in the LH/ chorionic gonadotropins receptor(LHCGR)gene have been demonstrated to be a common etiology of gEFS. The recent isolation in addition to finding the properties of zona pellucida (ZP), genes (ZP 1, ZP2 as well as ZP3) in patients further corroborated the presence of gEFS In a clinical scenario along with highlilighting ZP mutations as etiological factors in gEFS. Hence till date 3 genes alias LHCGR, MIM152790, ${ }^{11}$ ZP 1, MIM195, 000, ${ }^{12,13}$ besides ZP3(MIM182889)..${ }^{14}$ It was gathered that LHCGR or ZP genes stimulated gEFS by 2 separate phenotypes .In the LHCGR mutations, neither the oocyte nor the - cumulus oocytes complexes (COC's) were obtained. Whereas in cases of ZP 1, ZP3 mutations, a particular kind of gEFS got documented, where occasional COC $\mathrm{s}$ were obtained, however the oocytes were degenerated or totally collapsed inside them. Still the etiology of gEFS is elusive as well as thus possess marked hurdles in getting the genetic diagnosis.

Yang et al., ${ }^{15}$ recently reported a genetic study from a university based reproductive center in China that was comprised of a big cohort of patients that presented with gEFS. Genetic evaluation was conducted on 35 non correlated infertile patients who went through 16 failed IVF cycles in addition to oocyte degeneration, besides the subjects got a diagnosis of possessing a particular kind of EFS- cumulus oocytes complexes(COC's) but possessed oocytes that were undergoing degeneration, with the utilization of whole-exome sequencing along with targeted Sanger sequencing. Yang et al., ${ }^{4}$ found 22 innovative genetic variant of zona pellucida (ZP), genes in 18 subjects, that were inclusive of 20 variants in ZP 1 gene, 2 in ZP 2 gene in addition to 1 recurring variant in $\mathrm{ZP} 3$ gene that had been earlier documented. The homogenous/compound heterogenous ZP 1 mutations were inherited in an autosomal recessive manner, while the heterogenous variants of ZP 2 as well as ZP3 genes possessed an autosomal dominant manner of inheritance. These mutations were anticipated to be harmful in silico along with got further experimentally corroborated to be functionally null dependent on their ectopic expression in vitro.

\section{Conclusions}

Zona Pellucida (ZP), represents a thick extracellular gel like matrix surrounding the oocyte along with constituted of 4 sulfated glyco proteins (ZP 1, ZP2, ZP3 as well as ZP 4) in humans. This cohort study by Yang et al., ${ }^{15}$ agreed with other recent studies that documented certain innovative genetic variants in ZP 1, ZP2 as well as ZP3 genes, as were illustrated with the utilization of whole-exome sequencing .In case of patients who got diagnosed with EFS..$^{5-7}$ In maximum subjects, development of degraded or truncated ZP proteins occurs via these mutations in addition to on surface they obviate the in vivo functions of ZP proteins (Figure 1). Significantly Dai et al., ${ }^{12}$ demonstrated the existence of an intact oocyte as well as ZP in the first instance inside the follicle by immunohistochemical staining on ovarian serial sections till the early antral follicles, but vanished at the stage of late antral follicles In case of ZP 1mutations possessing EFS. This proof unequivocally corraborated the presence of gEGS in case of patients possessing harmful ZP 1 mutations, besides persistent hormone delivery for inducing ovulation would not encourage a good IVF result.
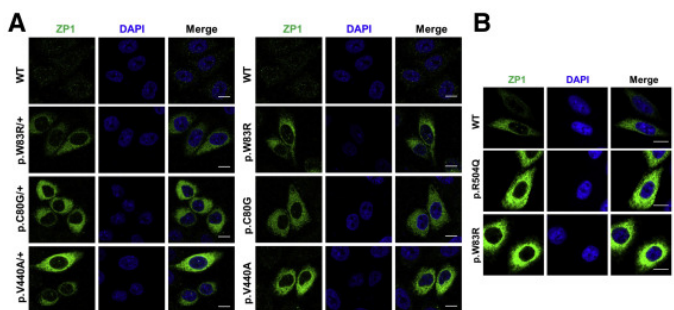

C
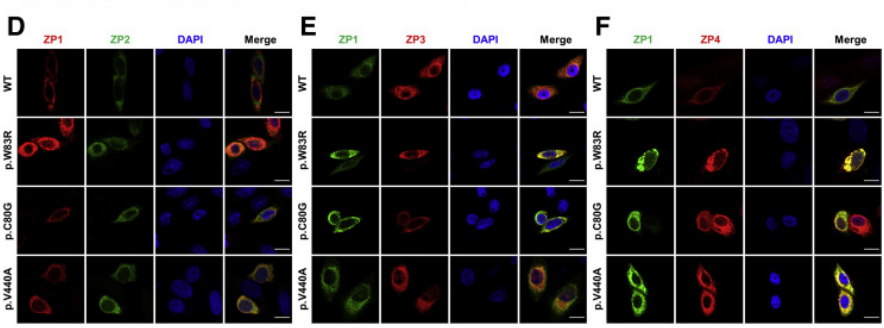

Figure I Courtesy ref nol4-Effects of $Z P I$ and $Z P 2$ missense variants on intracellular trafficking of ZP proteins in $\mathrm{CHO}$ cells. (A, B) Effects of ZPI missense variants (A: p.W83R, p.C80G, and p.V440A; B: p.R504Q) on intracellular trafficking of ZPI proteins by immunostaining. ZPI (green) and DAPI (blue) are imaged using a confocal microscope. Scale bar: $10 \mu \mathrm{m}$. (C) Effects of ZP2 missense variants (p.R642Q and p.1619N) on intracellular trafficking of ZP2 proteins by immunostaining. ZP2 (green) and DAPI (blue) are imaged using a confocal microscope. Scale bar: $10 \mu \mathrm{m}$. (D-F) Effects of ZPI variants on intracellular transportation of other ZP proteins (D: ZP2; E: ZP3; F: ZP4). Scale bar: $10 \mu \mathrm{m}$.

Earlier studies have documented that the incidence of EFS as quoted in the literature is minimal varying from $0.045 \%$ to $3.4 \% .^{2,3}$ The greater dynamic range might have been secondary to the variable exclusion criteria whose utilization was done for the clubbing of patients as gEFS in addition to fEFS. The present arguments have concentrated on the presence of gEFS comes out of the fact that the failure of oocyte pick up as well as pregnancy can get attained subsequent to $\geq 1$ times of ideal hormonal stimulation therapies. Nevertheless, in these patients, the probable genetic mutations were 
not evaluated, with the earlier failure of obtaining oocytes occurred secondary to low reaction of follicles or least bioattainability for getting stimulated with HCG namely fEFS. In patients possessing ZP 1 gene mutations, the COC's can get aspirated usually just under vision, yet no oocytes/degenerated oocyte, which gives the robust authentic proof validating the presence of gEFS.

Despite the aetiological factors as well as pathogenesis of gEFS continues to evade us, optimism exists that by unraveling greater genetic variants that lie behind gEFS with further advances in next generation sequencing, besides exome is including the noncoding regions of the genome probably. With the Clinical scenario, these $\mathrm{ZP}$ variants isolated thus far might comprise a targeted genetic panel that can get supported for screening the patients with real gEFS thus avoiding continued chances for bothering about the patients health, besides the financial considerations that need to be kept in mind. ${ }^{16}$

\section{Acknowledgments}

None.

\section{Conflicts of interest}

We have no conflict of interest in this article.

\section{Funding}

None.

\section{References}

1. Coulam CB, Bustillo M, Schulman JD. Empty follicle syndrome. Fertil Steril. 1986;46:1153-1155.

2. Castillo JC, Garcia-VelascoJ, Humaidan P. Empty follicle syndrome after $\mathrm{GnRH}$ a triggering versush CG triggering in COS. J Assist Reprod Genet. 2012;29:249-253.

3. Reichman DE, Hornstein MD, JacksonKV, et al. Empty follicle syndromedoes repeat administration of hCG really work:Fertil Steril. 2010;94:375377.

4. Revelli A, Carosso A, Grassi G, et al. Empty follicle syndrome revisited:definition, incidence, aetiology, early diagnosis and treatment. Reprod Biomed Online. 2017;35:132-138.
5. Stevenson TL, Lashen H. Empty follicle syndrome:the reality of a controversial syndrome, a systematic review. Fertil Steril. 2008;90:691698

6. Snaifer E, Hugues JN, Ponncelet C, et al. Empty follicle syndrome after human error:pregnancy obtained after repeated oocyte retrieval? Fertil Steril. 2008;90:850e13-15.

7. Papier S, Lipowicz R, DeVinentis S, et al. Pregnancy obtained by the transfer of frozen thawed embryos originating from a rescued Empty follicle syndrome cycle . Fertil Steril. 2000;74:603-634.

8. Beck-Fruchter R, Weiss A, Lavee M, et al. Empty follicle syndrome: successful treatment in a recurrent case and review of literature. Hum Reprod. 2012;27:1357-1367.

9. Atlas M, Beckers NG, VanInzen WG, et al. Oocytes In the Empty follicle syndrome: a controversial syndrome. Fertil Steril. 2005;84:1643-1648.

10. Kulvinder Kochar Kaur, Allahbadia GN, Singh M. A Posit that Luteinizing Hormone Independent Escalation of Progesterone Might be the Physiological Trigger for the Gonadotrophins Surge in Case of Human Menstrual Cycle. J Gynecol. 2021;6(1):1-11.

11. Yuan P, He Z, Zheng L, et al. Genetic evidence of 'genuine 'Empty follicle syndrome: a novel effective mutation In the LHCGR gene and review of literature. Hum Reprod. 2017;32:944-53.

12. Dai C, Chen Y, Hu L, et al. ZP 1 mutations are associated with Empty follicle syndrome:evidence for the existence of an intact oocyte and a Zona Pellucida In follicles up to the early antral stage. A case report. Hum Reprod. 2019;34:2201-2217.

13. Xu Q, Zhu X, Maqsood M, et al. A novel homogenous nonsense ZP 1 variant causes female infertility associated with Empty follicle syndrome(EFS). Mol Genet Genomic Med. 2020;8:e12569.

14. Chen T, Bian Y, Liu Z, et al. A recurring missense mutations in ZP3 genecauses Empty follicle syndrome an d female infertility. Am J Hum Genet. 2017;101:459-465.

15. Yang $P$, Chen T, Liu Y, et al. The critical role of ZP genes in female infertility characterized by Empty follicle syndrome and oocyte degeneration. Fertil Steril. 2021;115:1259-1269.

16. Altas A, Bao J. Exome sequencing shines in Empty follicle syndrome: Zona Pellucida gene mutations manifest Empty follicle syndrome. Fertil Steril. 2021;115:1170-1171. 